\title{
The effect of hip joint strengthening exercise using proprioceptive neuromuscular facilitation on balance, sit to stand and walking ability in a person with traumatic brain injury: a case report
}

\author{
Du Kyo Junga, Yijung Chung ${ }^{b}$ \\ ${ }^{a}$ Department of Physical Therapy, Soon Chun Hyang University Seoul Hospital, Seoul, Republic of Korea \\ ${ }^{\mathrm{b}}$ Department of Physical Therapy, College of Health Science and Social Welfare, Sahmyook University, Seoul, Republic of Korea
}

Objective: The purpose of this study was to investigate the effect of the hip joint strengthening exercises using proprioceptive neuromuscular facilitation (PNF) on the clinical symptoms and the treatment effects in balance, sit to stand, and gait abilities in patients with TBI.

Design: A single case study.

Methods: A 13-year-old adolescent with quadriplegia and hip joint control impairment participated in this four-week training intervention. The patient, diagnosed with TBI, wastreated with hip joint strengthening exercises using PNF. In the first week, we focused on strengthening the body, relaxing the hip flexors and activating the hip extensor muscles in order to solve the patient's physical function and body structure. From the 2nd and 4th week, we improved the motivation through the task-oriented method, and then weight-bearing training of the right lower extremity was proceeded by kicking a soccor ball with the left lower extremity. The exercises were performed for 4 weeks, 5 days a week, for 60 minutes with the exercise intensity gradually increased according to the subject's physical abilities.

Results: As a result of the study, the patient demonstrated improvements in the physical examination, which were evaluated before and after intervention and included the manual muscle test, modified Ashworth scale, sensory assessment, coordination assessment, Berg balance scale, 5-time sit to stand test, and the 10 meters walk test.

Conclusions: The results of this case suggest that a hip joint strengthening exercise program using PNF may improve hip control ability, balance, sit to stand and gait ability in a patient with TBI.

Key Words: Gait, Proprioceptive neuromuscular facilitation, Traumatic brain injury

\section{Introduction}

Traumatic brain injury (TBI) occurs by an accidental or intentional beating of the head and may be caused by car accidents, falls, or damage through sport activities [1]. Male children are more likely to suffer injuries than female children and are most likely to have TBI in the adolescent years between the ages 15 and 19 .
Children with TBI experience many problems of the body structure and function, such as muscle building, paralysis, weakness, mobilization difficulties, and spasticity due to increased muscle tone, and these problems can adversely affect the speed of normal operation and speech [2]. Also, there were many changes balance and gait ability, particularly in step length and gait velocity, of normal children and children with TBI [3]. Due to the limitations of activity and

Received: 15 May, 2017 Revised: 12 June, 2017 Accepted: 13 June, 2017

Corresponding author: Yijung Chung

Department of Physical Therapy, College of Health Science and Social Welfare, Sahmyook University, 815 Hwarang-ro, Nowon-gu, Seoul 01795, Republic of Korea

Tel: 82-2-3399-1637 Fax: 82-2-3399-1639 E-mail: yijung36@syu.ac.kr

(c) This is an Open-Access article distributed under the terms of the Creative Commons Attribution Non-Commercial License (http://creativecommons.org/licens es/by-nc/4.0) which permits unrestricted non-commercial use, distribution, and reproduction in any medium, provided the original work is properly cited.

Copyright @ 2017 Korean Academy of Physical Therapy Rehabilitation Science 
participation, children with TBI are reluctant to move and subsequently there is a risk of deterioration of physical strength and endurance; however, if treated with activities that the child enjoys, it can help to improve the level of physical strength [1].

Most children with TBI easily damage the frontal and temporal lobes due to shear forces, therefore, they need to focus on behavioral issues such as emotion and cognition during exercise [4]. These problems can have distracting, hyperactive, auditory, and visual problems that are not enough to filter out unnecessary sensory stimuli [5], and overall, the memory and intelligence abilities of TBI children may be less than before the impairment [6]. Various behavioral problems range from mere insecurity and psychotic behavior, however, rather than physical deficits, the changes in cognition, behavior, and personality has a greater effect on family dysfunction and stress levels.

Although support for the body against gravity occurs mainly in the sagittal plane in order to push and move the body forward [7], in a three-dimensional analysis, it can be seen that significant amount of work in the hip joint occurs in the frontal plane in controlling the pelvis and trunk against gravitational forces [8]. Recent mathematical models have shown that the gluteus medius plays an important role during the single support time and forward propulsion during the stance phase [9].

Proprioceptive neuromuscular facilitation (PNF) uses basic procedures, techniques, patterns, and philosophies to stimulate muscles and nerves and can be used as a way to promote muscular function, nervous function, social activities, as well as functional activities [10]. When the patient is standing or walking, many of the basic techniques and procedures of PNF can be used, and when resistance to posture and strong gait movement occurs, stimulation for muscle contraction occurs in the contralateral lower extremity and weakened trunk [10]. Allowing the patient to walk or stand as independently as possible allows for the patient to make self-corrections to dysfunctional movements, and can be used as an independent gait and resistance gait training. Although there are many studies based on use of PNF on patient with neuromuscular damage, there are currently no studies on the use of PNF intervention in children with TBI. Most of the existing papers have positively influenced the results of activities and participation by solving problems of body function and body structure.

In the international classification of dysfunction (ICF), physical function, structure, activity, and participation are components of function, and disability is classified. There is a complex interaction between these factors. Changes in one element have the potential to change other elements, and these interactions can act in both directions to change the health condition itself. The factors related to background factors (positive family support, medical equipment support), and personal factors (motivation) may have a positive effect on the patient's activities and participation.

This study focused on the environmental, individual, and background factors that created patient motivation and were used to solve the body function and structural problem. The factor to this problem was due to the lack of cognitive ability, lack of motivation, and depression in the patient, and therefore disallowed the patient to actively participate in the treatment sessions. Therefore, in order to promote activities and participation, the philosophy of PNF was used to find out the potential of the patient through a positive and functional approach and a holistic approach was applied that considers not only the environmental aspects but also personal tendencies.

Therefore, the purpose of this study was to investigate the effects of hip strengthening exercises with PNF techniques on balance, sit to stand, and gait abilities in children with TBI.

\section{Methods}

\section{Subjects}

This study included one patient who was admitted and diagnosed with TBI at Soon Chun Hyang University Seoul Hospital was enrolled for the study for four weeks from April 25, 2016 to May 18, 2016. The subject provided their voluntary consent after being informed of the purpose and details of the study (IRB No. 2017-03-001-001).

Onset of injury occurred in January 9, 2016. The 13-yearold male patient had a height of $164 \mathrm{~cm}$, weight of $44 \mathrm{~kg}$, and no previous medical history. The patient's mini mental state examination score was 14 and although consciousness was clear, the patient showed problems with cognitive function and showed comprehension, orientation due to post-traumatic stress, psychiatric examination was also performed. For the hand function test, the right grip was $15 \mathrm{~kg}$ and the outer grip was $1.5 \mathrm{~kg}$, hand grip was $0.5 \mathrm{~kg}$, left grip was 16 $\mathrm{kg}$, outer grip was $2 \mathrm{~kg}$, and the palm grip was $2.2 \mathrm{~kg}$ and so the left hand function was superior to the right hand, and the right finger showed impairment The 2-point discrimination was impaired on the right hand. For the hand coordination 
Table 1. General characteristics of subject

\begin{tabular}{lc}
\hline Characteristic & Value \\
\hline Gender & Male \\
Age (y) & 13 \\
Onset & 2016.1 \\
MMSE & 14 \\
TUG (s) & 46.57 \\
MBI & 52 \\
\hline
\end{tabular}

MMSE: mini-mental state examination, TUG: timed up and go, MBI: modified Barthel index.

test, the Jebsen-Taylor hand function Test showed that that there was a problem with hand coordination as demonstrated by the scores 0 for the right hand and 17 for the left hand. Since the subject experienced difficulty with reading and writing, the writing assessment could not be conducted (Table 1).

The modified Barthel index, which is an evaluation of activities of daily living, was 52 points. In the sitting position, after establishing trunk alignment, muscles that perform right lateral trunk flexion were stiff. In the manual muscle test (MMT), the muscle strength of the trunk extensor was a $2+$, right hip flexor was a 3 , left hip flexor was a $3+$, right knee joint flexor was a 3 , left knee joint flexor was a $3+$, right dorsiflexor was a 3-, left dorsiflexor was a 3, and in particular, the right hip joint extensor muscle and abductor was a $2-$. In addition, muscle tension was scored as a G1 + of the hip joint flexor and abductor muscles on the modified Ashworth scale (MAS). For sensory evaluation, the superficial sensation (light touch) and deep sensation (proprioception) tests were performed on the right ankle and plantar surface and toes, which exhibited sensory impairment. For the coordination test, the toe to therapist finger was performed. For the Berg balance scale (BBS), it was possible to maintain an independent balance in sitting position, but had difficulty in maintaining static and dynamic standing balance and showed a tendency to fall back immediately when assistance was removed. The subject required minimum and moderate assistance level for sitting and standing and walking was possible when minimal support was provided. For the 10 meters walk test (MWT), it took 20.31 seconds to walk under supervision using a walker and in a supervised timed up and go test, difficulties in standing, turning, and sitting from a standing position were exhibited with a score of 46.57 seconds (Table 1 ).

\section{Procedures}

\section{Research equipment}

\section{Physical examination}

In order to perform a physical examination, the MMT was used to assess for strength, the MAS was used to assess spasticity and tension, a sensory assessment was used to assess sensation of the feet, and the coordination assessment was performed to evaluate the body function and structure of the subject. The strength test is used to assess the degree of muscle strength using gravity and resistance provided by the examiner [11]. Muscle spasticity and hypertonicity can be assessed using the MAS to assess for stiffness and tension and also has high inter-rater reliability [12]. Proprioception sensation evaluates the ability to sense the position of the joint, moves the range of motion of the joint to be evaluated to maintain a static posture and to assume the same position with the opposite body part [13].

\section{Berg balance scale}

The BBS is composed of 14 balance items based on activities of daily living and is commonly used to measure balance ability of the elderly population. This can be used as a tool for evaluating static and dynamic balance ability of elderly stroke patients in predicting falls and is highly sensitive to detecting the degree of recovery [14].

\section{5-time sit to stand test}

The sit to stand test was performed 5 times to measure lower extremity strength. This method was used to measure the time it takes to perform 5 repetitions of standing up and sitting in a chair without a backrest and armrests and reported a high inter-rater reliability intra-class correlation coefficient $=0.87[15]$.

\section{0 meters walk test}

A 10MWT was used to determine the change in walking speed. During the 10 meters walk, the subject was trained to walk comfortably and was assessed during the middle 6 meters to eliminate the effect of $2 \mathrm{M}$ deceleration and $2 \mathrm{M}$ acceleration zones [16]. The inter-rater reliability was reported to be high $(\mathrm{r}=0.89-1.00)$ [17].

\section{Treatment method}

Proprioceptive neuromuscular stimulation [10] was performed on the subject diagnosed with TBI by the following 
exercise method.

\section{Weeks 1 to 4}

In the first week, we focused on strengthening the body, relaxing the hip flexors and activating the hip extensor muscles in order to solve the patient's physical function and body structure. From the 2 nd and 4 th week, we improved the motivation through the task-oriented method, and then the body function and structure were focused again. The first exercise method was based on the philosophy of PNF, using the strong left side of the patient in order to increase the strength of the body flexor muscles, scapular anterior depression pattern and pelvic anterior elevation patterns were used to roll forward and a combination of isotonic were applied. For the second method, in order to increase the lower trunk muscle strength, the contraction relaxation technique while lying supine and respiration was used to increase the body flexion range during the contract-relax application and to increase the strength of the lower body, a combination of isotonic was applied using bilateral lower extremity flexion with a knee flexion pattern to bend the knee.

For the third method, to increase the hip flexion range of motion, and to increased hip range of motion, hip flexion adduction with external rotation and knee flexion pattern while side-lying and after applying the contract-relax technique, in order to increase hip flexor muscle strength, hip flexion, abduction, and internal rotation with knee extension pattern was used with the timing for emphasis and combination of isotonic applied. For the fourth method, the pelvic anterior elevation and pelvic posterior depression pattern was used to increase hip joint muscle strength and a combination of isotonic and stabilizing reversals were applied.

\section{2nd to 4th weeks}

As a result of 1 week of treatment, the patient's endurance and motivation problems allowed the patient to be more activated and motivated and using a likeable activity, such as soccer, was considered as a more functional approach. For the fifth exercise, based on exercise control and learning, the forward pelvic pattern and respiration were performed in a sitting position, and stabilizing reversals were applied to the scapula and pelvis using the forward and backward patterns. For the sixth method, a combination of isotonic of the hip joint was applied based on exercise control and learning. For the seventh method, based on the functional approach which is the philosophy of PNF, a combination of isotonic was applied using practical functional movements while moving from a standing position to a sitting position in order to emphasize the exoskeleton of the hip joint. For the eighth method, to stabilize the trunk and to improve the weight support ability of the right lower limb, and after establishing a posterior tilted pelvis in standing posture, a combination of isotonic was applied while the weight of the right lower extremity was supported and the left foot was moved forward to improve walking ability after sufficient weight support training. For the ninth method, in order to promote more weight-bearing capacity and motivation of the right foot using more realistic task, such as soccer, after weight shifting to the right, the exercise that consisted of controlling the movement of a ball forward and backward was performed. For the tenth method, a soccer ball was used where the therapist rolled the ball to the subject while the patient caught the ball, and by having the subject kick the ball into the goalpost, this showed to be a practical task in improving the subject's weight-bearing ability on the right side. Patients sometime showed endurance difficulties and promoted patient motivation through their favorite soccer to promote patient motivation. Finally, in order to improve the walking ability, weight training to the right was performed, followed by training to the left, and gait training while adjusting the ball using a soccer ball and practical gait training was repeated. In order to improve the patient's potential ability using the philosophical mobility storage, after the treatment time, the subject was given the task of lifting the buttocks and when the patient was viewed as a whole based on their environmental and personal characteristics, the subject could not use the time efficiently after the treatment time or on the weekend. The mother of the subject was asked to encourage the subject to practice increasing the left foot step length. Based on exercise control and motor learning, mobility was improved in the early stages of treatment and improved stability in the second and the third was to improve mobility and stability at the same time. Finally, practical gait training was practiced with active motivation of the patient in a practical environment. From the low position to the actual gait training, the hip extensor and abductor muscles and were repeatedly contracted through repeated extension and abduction movements (Figure 1).

In each posture, the basic PNF procedures, techniques, and philosophy were used. The above 11 exercises and home exercises were performed for 4 weeks, 5 days a week, for 60 minutes with the exercise intensity gradually increased according to the subject's physical abilities, and to improve jumping ability and motivation, the patient's favorite dance 


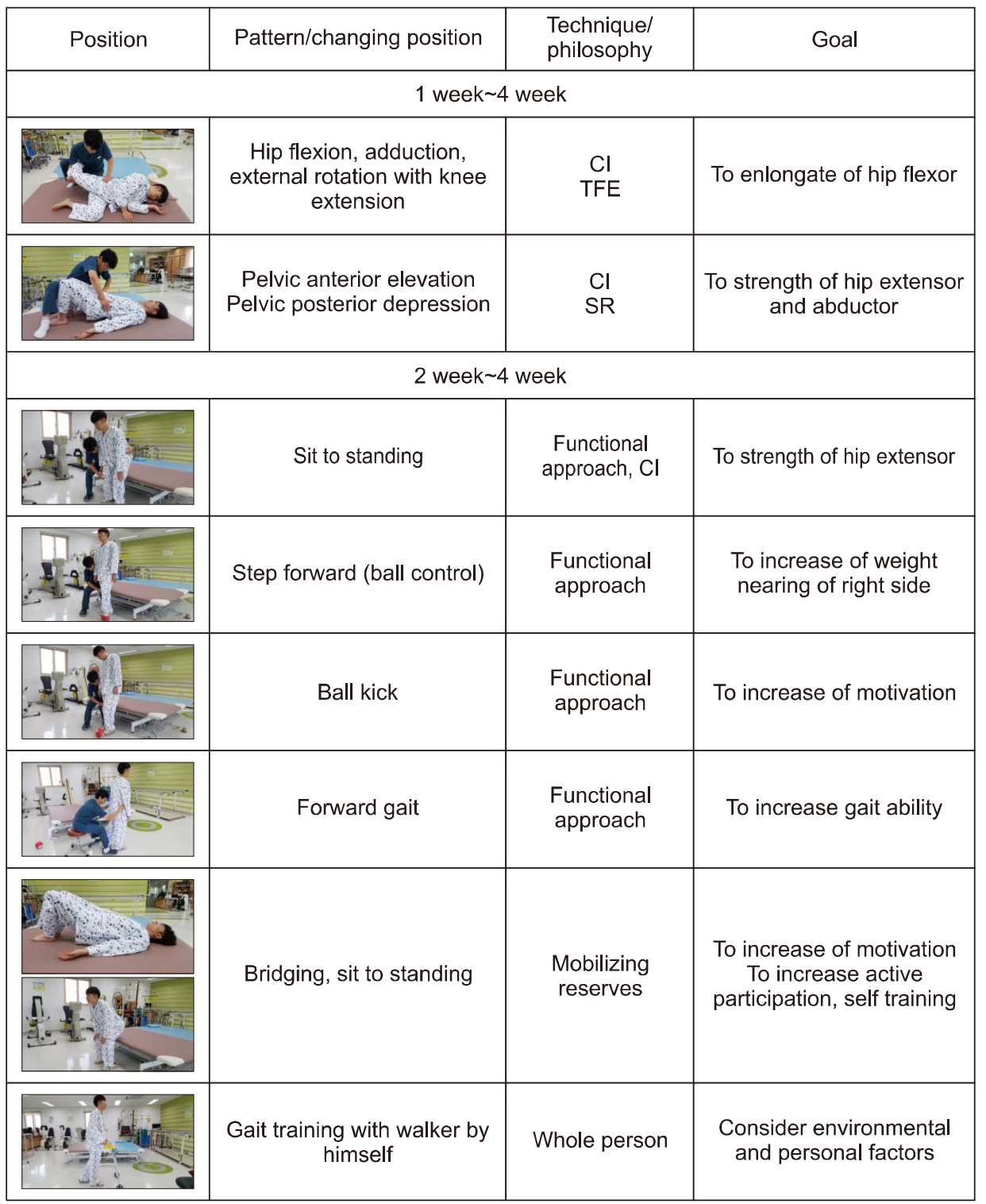

Figure 1. Intervention for 4 weeks. CI: combination of isotonic, TFE: timing for emphasis, SR: stabilizing reversals. was used. In the first week of the training period, in order to solve the physical function and structural problem and patient's lack of endurance and motivation, the activity in which the subject enjoyed, such as soccer, was used as a participation method. When motivation increased, the patient moved to a lower posture again to resolved the physical function and structural problem. The exercise was applied according to the psychological stage of the patient for 4 weeks. In addition, the bike was ridden for 40 minutes in the exercise room and functional electrical stimulation (FES) was applied to the right ankle joint where dorsiflexion occurs for 20 minutes. Upper extremity training was performed afterwards in the occupational therapy room for 30 minutes.

\section{Data and statistical analysis}

For this study, in order to investigate the changes in physical examination, sit to stand, balance after intervention, the results of the subject before and after the experiment using the result and mean values were compared at each time point to determine the changes in gait ability. In addition, the difference before and after treatment was assessed as a percent change (\%improvement $=$ (pre-treatment assessment-posttreatment assessment/pre-treatment evaluation $\times 100$ ). 
Table 2. Changes of lower extremity (clinical test) before and after intervention

\begin{tabular}{lccccc}
\hline \multirow{2}{*}{ Lower extremity (clinical test) } & \multicolumn{2}{c}{ Right lower extremity } & \multicolumn{2}{c}{ Left lower extremity } \\
\cline { 2 - 3 } \cline { 5 - 5 } & Before-intervention & After-intervention & Before-intervention & After-intervention \\
\hline Hip extensor (MMT) & $2-$ & $3+$ & 3 & $2+$ & 4 \\
Hip abductor (MMT) & $2-$ & 0 & $2+$ & 4 \\
Hip flexor (MAS) & $1+$ & 0 & 1 & 0 \\
Hip adductor (MAS) & 4 & 1 & 1 & 0 \\
Thigh (LT) & 4 & 1 & 1 & 1 \\
Sole of foot (LT) & 4 & 1 & 1 & 1 \\
Ankle (proprioception) & 4 & 5 & 1 & 1 \\
Toe (proprioception) & 3 & 5 & 5 \\
Toe to therapist finger (coordination) & & 1 & 1 \\
\hline
\end{tabular}

MMT: manual muscle testing, MAS: modified Ashworth scale, LT: light touch.

\section{Results}

\section{Changes in physical examination}

\section{Changes in muscle strength}

The MMT before and after the hip joint strengthening exercise using the proprioceptive neuromuscular stimulation method were as follows (Table 2). The strength test for before and after the hip joint strengthening exercise improved from a $2-$ to a $3+$ for the right hip extensor muscles, a $2-$ to 3 for the hip abductor muscles, from a $2+$ to 4 for the left hip abductor muscle (Table 2).

\section{Changes in muscle tone}

In the MAS, there was a decrease in muscle tone from 0 to 1 in the right hip flexor muscle and 0 to 1 in the hip joint muscle group before and after intervention and there was a decrease in muscle tone of the left hip flexor muscle from 1 to 0 and from a 1 to 0 in the hip adductor muscle (Table 2).

\section{Assessment of sensory changes}

In the sensory evaluation, light touch was improved from 4 to 1 in the right thigh before and after intervention and in the soles of the feet before and after intervention and slight tactile sensation on the left thigh and soles of the feet by 1 point (Table 2). Before and after intervention, proprioception was improved from 4 to 1 in the right ankle joint and toe and the left ankle joint and toe improved by 1 point (Table 2 ).

\section{Changes in coordination}

In the toe-to-therapist finger to toe coordination test, there was an improvement from 3 to 5 in the right lower limb before and after intervention, and an improvement of 5 points

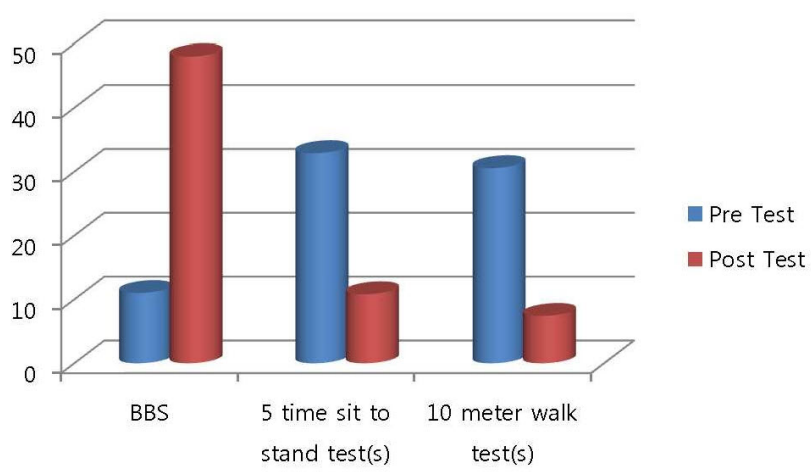

Figure 2. Pre-test and post-test of BBS, 5-time sit to stand, 10 meters walk test. BBS: Berg balance scale.

in the left lower extremity (Table 2).

\section{Comparison of the 5-time sit to stand test}

The results of the five time sit-to-stand test were as follows (Table 3). On the fifth test, scores improved from 32.91 to 10.81 seconds after intervention (Figure 2).

\section{Comparison of the 10 meters walk test}

The 10MWT results before and after intervention were as follows (Table 3). The 10MWT scores improved from 30.56 seconds before intervention to 7.41 seconds after intervention (Figure 2).

\section{Comparison of the Berg balance scale scores}

The changes in BBS scores are as follows (Table 4). Sitto-stand was improved from 1 to 4 points after intervention, standing without support improved from 3 to 4 points after intervention, and maintaining sitting position without back 
Table 3. Changes of 5-time sit to stand test and 10 meters walk test before and after intervention

\begin{tabular}{ccc}
\hline Task & Before-intervention & After-intervention (\% improvement) \\
\hline 5-time sit to stand test (s) & 32.91 & $10.81(67 \%)$ \\
10 meters walk test (s) & 30.56 & $7.41(75 \%)$ \\
\hline
\end{tabular}

Table 4. Changes of Berg balance scale before and after intervention

\begin{tabular}{lcc}
\multicolumn{1}{c}{ Task } & Before-intervention & After-intervention (\% improvement) \\
\hline Sitting to standing & 1 & 4 \\
Standing unsupported & 3 & 4 \\
Sitting unsupported & 4 & 4 \\
Standing to sitting & 1 & 4 \\
Transfers & 1 & 4 \\
Standing with eyes closed & 1 & 4 \\
Standing with feet together & 0 & 3 \\
Reaching forward with outstretched arm & 0 & 3 \\
Retrieving object from floor & 0 & 4 \\
Turning to look behind & 0 & 3 \\
Turning 360 degrees & 0 & 3 \\
Placing alternate foot on stool & 0 & 3 \\
Standing with one foot in front & 0 & 1 \\
Standing on one foot & 0 & $48 / 56(336 \%)$ \\
Berg balance scale total & $11 / 56$ & 4 \\
\hline
\end{tabular}

support was a score of 4 prior to and after intervention.

Stand-to-sit ability was improved from 1 to 4 and movement from chair to chair was improved from 1 to 4 after intervention. The ability to stand without support and with eyes closed improved from 1 to 4 after intervention. The subject's ability to stand with two feet together improved from 0 to 4, and standing with arms reached forward improved from 0 to 3 after intervention. The ability to pick up objects from the floor increased from 0 to 3 after intervention. The ability to look back toward the left and right improved from 0 to 4 , and the ability to rotate 360 degrees in place improved from 0 to 3 points after intervention. The ability to place one foot on top of the other at an equal distance in height improved from 0 to 3 after intervention. Tandem foot standing ability improved from 0 to 3 , and the ability to stand on one leg increased from 0 to 1 after intervention. The total BBS score increased from 11 to 48 points.

\section{Discussion}

The purpose of this study was to investigate the effect of performing hip strengthening exercises using PNF techniques in a patient with $\mathrm{TBI}$ and to investigate the clinical fea- tures and treatment effects that are associated with balance and gait. Symptoms of motor impairment in children with TBI, such as delayed movement, lead to long-term disability of exercise skills [18]. Loss of exercise control ability to perform delicate functions may be due to asymmetrical posture, balance reaction impairment, and decreased walking ability [19].

The gluteus medius is the largest of the abductor muscles and occupies a cross-sectional area of approximately $60 \%$ [20]. In order to stand on one leg, the hip abductor muscles need to provide support [21], and the gluteus medius plays a main role during the single support time phase [22]. Therefore, selective muscle strengthening exercises of the hip abductor muscles are important in making functional lower extremity movements and plays an important role in regulating the dynamic stability of the lateral pelvis [23].

Although weakness of the hip muscles were observed in this study, the relaxation techniques applied onto the hip flexors worked to lower muscle tension and improve hip flexor and abductor muscle strength. High intensity training was performed to elicit the potential of the patient during treatment since recent reports have suggested that patients with TBI may benefit from high-intensity training treatment programs $[24,25]$. Study with twenty-two subjects with 
stroke and two patients with brain surgery underwent intensive repetition of exercise with PNF showed improvement in spontaneous movement of the injured limb of the subjects with brain injury [26]. After the PNF method was applied and gait was evaluated in the patients hemiplegia, it has been concluded that treatment with PNF allowed partial improvement of walking disorders performed in subjects. The mean walking speed of the patients improved from 0.44 to $0.59 \mathrm{~m} / \mathrm{s}$. The subject in this case also demonstrated problems with muscle strength and endurance and had endurance difficulties during the exercises. Children with severe head injury require intensive rehabilitation, and complex problems can have a negative impact on cognitive ability, skills, memory, language, and academic ability [27]. The strength and agility for children and adolescents with severe TBI for 4 years were found to have low levels of performance in exercise fitness, strength, agility, durability, and coordination which can limit the children's participation in sport and physical activity [28]. For children, it is important for work to be in a form of play and that the environment must be fun, rigorous, and motivating for the therapeutic training [29], and sensory play groups, which can be trained in developmental skills, should be motivating and enjoyable [30]. During this study, although the subject had become bored and had lost interest in the exercise, and exhibited endurance issues, however, a soccer ball was used to promote walking and balance ability and to create active interest in the treatment of the subject. The physical function and structural problem of the subject was attempted to be solved according to the treatment, activity and participation part of the international classification of functioning, disability and health model.

In this study, increased tension in the hip flexor and adductor muscles exhibited decreased balance and gait scores, decreased hip extensor and abductor muscle strength had a negative effect on balance and gait. After intervention, there was a positive effect on balance and gait as the tension in the hip flexor and adductor muscles decreased and increased hip extensor and abductor muscle strength showed a positive effect on balance and gait. In addition, although it was found that the ankle-strengthening exercise by the active PNF method in the acute phase of a TBI patient it is considered that hip strengthening exercises helped to improve strength, muscle tension, sensation, and coordination has led to improvement of hip and ankle control ability and compared to previous studies, it has been shown in this study that the hip strengthening exercise using the PNF method produced a positive effect on the subject. Decreased hip joint control ability appears to be correlated with balance and gait from a functional aspect and that the problem should be focused on strengthening the hip joint using the PNF method in order to improve hip joint control ability, balance, sitting up and walking ability.

The study is limited by the inclusion of one TBI patient, and since the PNF method was used along with multiple treatments such as occupation therapy, bicycle riding, and FES, it would be difficult to estimate the effect of the PNF method only.

\section{Conflict of Interest}

The authors declared no potential conflicts of interest with respect to the authorship and/or publication of this article.

\section{References}

1. Effgen SK. Meeting the physical therapy need of children. 2nd ed. Philadelphia: F.A. Davis Company; 2013.

2. Chaplin D, Deitz J, Jaffe KM. Motor performance in children after traumatic brain injury. Arch Phys Med Rehabil 1993;74: $161-4$.

3. Katz-Leurer M, Rotem H, Lewitus H, Keren O, Meyer S. Relationship between balance abilities and gait characteristics in children with post-traumatic brain injury. Brain Inj 2008;22: 153-9.

4. Kirkwood M, Janusz J, Yeates KO, Taylor HG, Wade SL, Stancin $\mathrm{T}$, et al. Prevalence and correlates of depressive symptoms following traumatic brain injuries in children. Child Neuropsychol 2000;6:195-208.

5. Fay GC, Jaffe KM, Polissar NL, Liao S, Rivara JB, Martin KM. Outcome of pediatric traumatic brain injury at three years: a cohort study. Arch Phys Med Rehabil 1994;75:733-41.

6. Bowen JM, Clark E, Bigler ED, Gardner M, Nilsson D, Gooch J, et al. Childhood traumatic brain injury: neuropsychological status at the time of hospital discharge. Dev Med Child Neurol 1997;39:17-25

7. Carr JH, Shepherd RB. Neurological rehabilitation: optimizing motor performance. 2nd ed. Edinburgh: Churchill Livingstone; 2010.

8. Eng JJ, Winter DA. Kinetic analysis of the lower limbs during walking: what information can be gained from a three-dimensional model? J Biomech 1995;28:753-8.

9. Liu MQ, Anderson FC, Pandy MG, Delp SL. Muscles that support the body also modulate forward progression during walking. J Biomech 2006;39:2623-30.

10. Adler SS, Beckers D, Buck M. PNF in practice: an illustrated guide. 3rd ed. Heidelberg: Springer; 2008.

11. Kendall FP, McCreary EK, Provance PG, Rodgers MM, Romani WA. Muscles: testing and function, with posture and pain. 5 th ed. 
Baltimore: Lippincott Williams and Wilkins; 2005.

12. Ansari NN, Naghdi S, Younesian P, Shayeghan M. Inter- and intrarater reliability of the Modified Modified Ashworth Scale in patients with knee extensor poststroke spasticity. Physiother Theory Pract 2008;24:205-13.

13. O'Sullivan SB, Schmitz TJ. Physical rehabilitation. 5th ed. Philadelphia: F.A. Davis; 2007.

14. Berg KO, Maki BE, Williams JI, Holliday PJ, Wood-Dauphinee SL. Clinical and laboratory measures of postural balance in an elderly population. Arch Phys Med Rehabil 1992;73:1073-80.

15. Lord SR, Murray SM, Chapman K, Munro B, Tiedemann A. Sit-to-stand performance depends on sensation, speed, balance, and psychological status in addition to strength in older people. J Gerontol A Biol Sci Med Sci 2002;57:M539-43.

16. Bohannon RW, Andrews AW, Thomas MW. Walking speed: reference values and correlates for older adults. J Orthop Sports Phys Ther 1996;24:86-90.

17. Steffen TM, Hacker TA, Mollinger L. Age- and gender-related test performance in community-dwelling elderly people: SixMinute Walk Test, Berg Balance Scale, Timed Up \& Go Test, and gait speeds. Phys Ther 2002;82:128-37.

18. Wallen MA, Mackay S, Duff SM, McCartney LC, O'flaherty SJ. Upper-limb function in Australian children with traumatic brain injury: A controlled, prospective study. Arch Phys Med Rehabil 2001;82:642-9.

19. Carr JH, Shepherd RB. Stroke rehabilitation: guidelines for exercise and training to optimize motor skill. Edinburgh: ButterworthHeinemann; 2003.

20. Clark JM, Haynor DR. Anatomy of the abductor muscles of the hip as studied by computed tomography. J Bone Joint Surg Am
1987;69:1021-31.

21. Neumann DA. Kinesiology of the musculoskeletal system. St. Louis: Mosby; 2002.

22. Dalstra M, Huiskes R. Load transfer across the pelvic bone. J Biomech 1995;28:715-24.

23. Schmitz RJ, Riemann BL, Thompson T. Gluteus medius activity during isometric closed-chain hip rotation. J Sport Rehabil 2002;11:179-88.

24. Canning CG, Shepherd RB, Carr JH, Alison JA, Wade L, White A. A randomized controlled trial of the effects of intensive sit-tostand training after recent traumatic brain injury on sit-to-stand performance. Clin Rehabil 2003;17:355-62.

25. Gajdosik CG. Ability of very young children to produce reliable isometric force measurements. Pediatr Phys Ther 2005; 17:251-7.

26. Kawahira K, Shimodozono M, Ogata A, Tanaka N. Addition of intensive repetition of facilitation exercise to multidisciplinary rehabilitation promotes motor functional recovery of the hemiplegic lower limb. J Rehabil Med 2004;36:159-64.

27. Massagli TL, Jaffe KM, Fay GC, Polissar NL, Liao S, Rivara JB. Neurobehavioral sequelae of severe pediatric traumatic brain injury: a cohort study. Arch Phys Med Rehabil 1996;77:223-31.

28. Rossi C, Sullivan SJ. Motor fitness in children and adolescents with traumatic brain injury. Arch Phys Med Rehabil 1996;77: 1062-5.

29. Parham LD, Fazio LS. Play in occupational therapy for children. 2nd ed. St. Louis: Mosby; 2008.

30. LaForme Fiss AC, Effgen SK, Page J, Shasby S. Effect of sensorimotor groups on gross motor acquisition for young children with Down syndrome. Pediatr Phys Ther 2009;21:158-66. 